\title{
Cortical excitability following passive movement
}

\author{
Hideaki ONISHI, PhD, $\mathrm{PT}^{1,2}$ \\ ${ }^{1)}$ Institute for Human Movement and Medical Sciences, Niigata University of Health and Welfare \\ ${ }^{2)}$ Department of Physical Therapy, Niigata University of Health and Welfare
}

\begin{abstract}
In brain injury rehabilitation, passive movement exercises are frequently used to maintain or improve mobility and range of motion. They can also induce beneficial and sustained neuroplastic changes. Neuroimaging studies have revealed that passive movements without motor commands activate not only the primary somatosensory cortex but also the primary motor cortex, supplementary motor area, and posterior parietal cortex as well as the secondary somatosensory cortex (S2) in healthy subjects. Repetitive passive movement has also been reported to induce increases or decreases in cortical excitability. In this review, we focused on the following: cortical activity following passive movement; cortical excitability during passive movement; and changes in cortical excitability after repetitive passive movement.
\end{abstract}

Key words: Passive movement, Cortical excitability, Magnetoencephalography, Transcranial magnetic stimulation

(Phys Ther Res 21: 23-32, 2018)

$\mathbf{V}$ arious cortical imaging techniques measure brain activity, including functional magnetic resonance imaging (fMRI), positron emission tomography (PET), near-infrared spectroscopy (NIRS), electroencephalography (EEG), magnetoencephalography (MEG), and transcranial magnetic stimulation (TMS). Because EEG and MEG have excellent temporal resolution compared to fMRI, PET, and NIRS, they have been used to analyze the temporal aspects of cortical sensorimotor information processing; MEG fields, in particular, provide a direct reflection of the primary neural current, giving excellent spatial and temporal resolution.

TMS is a noninvasive brain stimulation technique that can be used to determine corticospinal excitability by measuring the muscular response to stimulation (the motorevoked potential, MEP). Intracortical inhibition (ICI) can be examined by the paired-TMS paradigm, by presenting a subthreshold conditioning stimulus followed by a suprathreshold test stimulus. With a short inter-stimulus interval (ISI) of 1-4 ms, the test responses are inhibited, known as short interval intracortical inhibition (SICI) ${ }^{1)}$. This inhibi-

Received: August 6, 2018

Accepted: September 12, 2018

Advance Publication by J-STAGE: November 30, 2018

Correspondence to: Hideaki Onishi, Department of Physical Therapy, Niigata University of Health and Welfare, 1398 Shimami-cho, Kita$\mathrm{Ku}$, Niigata City, 950-3198, Japan

\# e-mail: onishi@nuhw.ac.jp

doi: 10.1298/ptr.R0001 tion depends on local gamma-aminobutyric acid A (GABAa) receptor-mediated cortical inhibition and so is used as an indicator of GABAa-mediated circuit activity in the primary motor cortex ${ }^{2-6)}$. Depending on the ISI between the nerve stimulus and TMS pulse, the peripheral nerve stimulation delivered prior to the TMS pulse either inhibits the MEP or facilitates $i^{7,8)}$. MEPs are suppressed by electrical stimulation with an ISI of approximately $20 \mathrm{~ms}$, which is the first cortical component of somatosensory evoked potential following peripheral nerve stimulation; this is referred to as short latency afferent inhibition (SAI) ${ }^{7,9-12)}$. Conversely, MEPs are facilitated by electrical stimulation with an ISI of about 50-100 ms between the electrical stimulation and TMS pulse, known as afferent facilitation $(\mathrm{AF})^{9,12-15)}$. Pharmacological studies have demonstrated that cholinergic and GABAergic systems both play a role in $\mathrm{SAI}^{16,17)}$

There have been several studies of cortical activities or cortical excitability following voluntary movement, passive movement, peripheral nerve stimulation, mechanical stimulation, motor-point stimulation, water immersion, and noninvasive transcranial electrical brain stimulation. These have used fMRI, PET, NIRS, EEG, MEG, and TMS and have involved SICI, SAI, and AF techniques. In this review, we focus on the cortical activity and cortical excitability that arise from passive movement. 
a) Active movement

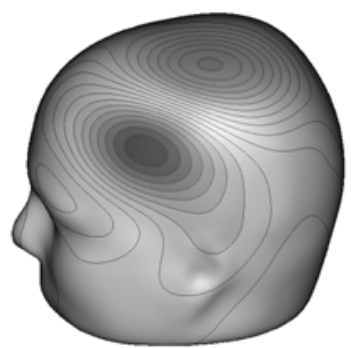

$34 \mathrm{~ms}$

b) Passive movement

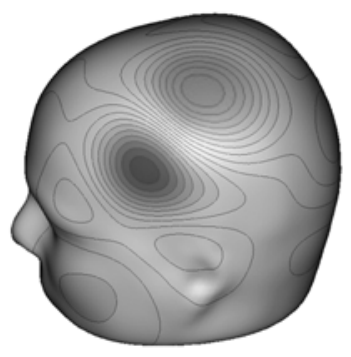

$34 \mathrm{~ms}$

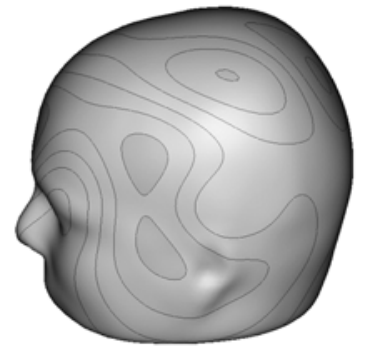

$89 \mathrm{~ms}$

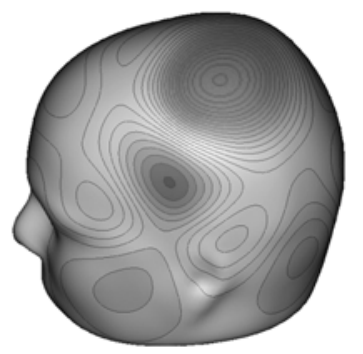

$89 \mathrm{~ms}$

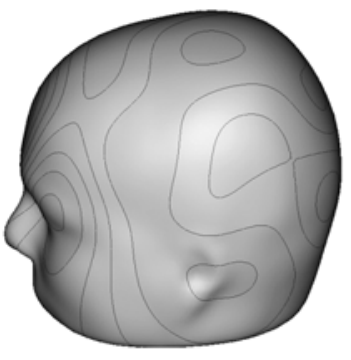

$121 \mathrm{~ms}$

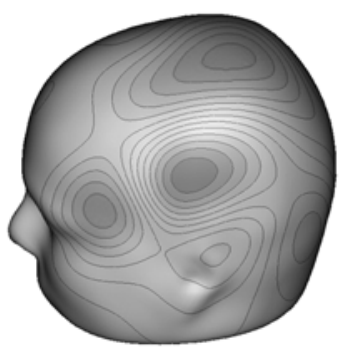

$121 \mathrm{~ms}$

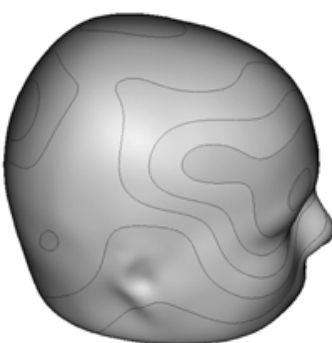

$140 \mathrm{~ms}$

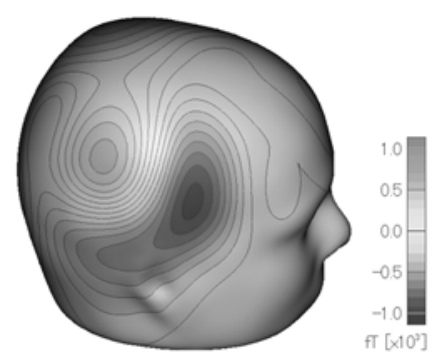

$140 \mathrm{~ms}$

Figure 1.

Isocontour maps over the left hemisphere at $34 \mathrm{~ms}, 89 \mathrm{~ms}, 121 \mathrm{~ms}$, and over the right hemisphere at $140 \mathrm{~ms}$, after active (a) and passive (b) movement in a representative subject. Red areas indicate magnetic flux exiting the head and blue areas flux entering the head. (Onishi et al., 201329) )

\section{Cortical Activation Following Passive Finger Movement}

Numerous studies have measured brain activity following passive movement using fMRI and PET, revealing that passive movements without motor commands activate not only the primary somatosensory cortex (S1), but also the primary motor area (M1), supplementary motor area (SMA), posterior parietal cortex (PPC), and bilateral secondary somatosensory areas $(\mathrm{S} 2)^{18-23)}$. However, the M1 activation in response to passive movement was not observed in patients with severe distal sensory neuropathy, suggesting that peripheral somatosensory afferent activation contributes to M1 activation ${ }^{19)}$. However, unlike MEG, PET, and fMRI do not have sufficient temporal resolution to elucidate the time course of activity in these cortical areas.

Some studies have used MEG systems to investigate the somatosensory evoked fields (SEFs) that accompany passive movement ${ }^{24-28)}$. For example, Xiang et al. ${ }^{28)}$ identified four SEF components, with peak latencies of 20, 46, 70 , and $119 \mathrm{~ms}$ after the onset of passive finger movement. Several researchers have reported that the large SEF component after passive movement was of a long duration, with two peaks from 30 to $100 \mathrm{~ms}$ after the onset ${ }^{24-26)}$. The equivalent current dipoles (ECDs) of these two components were located in area $3 b^{24)}$, area $4^{25)}$, and areas $3 \mathrm{~b}$ and $4^{26,28)}$. However, many MEG studies have shown no evidence of activity in the SMA, PPC, or S2 following passive move- ments. In a previous study, we recorded SEFs following active and passive finger movement with a multiple dipole analysis system to examine the detailed time course of cortical activity and source localizations ${ }^{29}$. Consistent with earlier studies, the two peaks of the MEG response associated with passive movement were recorded between 30 and 100 ms after the onset of movement. Figure 1 shows the resulting isocontour maps over the left hemisphere at $34 \mathrm{~ms}, 89$ $\mathrm{ms}$, and $121 \mathrm{~ms}$, and over the right hemisphere at $140 \mathrm{~ms}$, after active and passive movements. The earliest and second components (PM1 and PM2) had peaks at approximately 36 and $86 \mathrm{~ms}$ after passive movement onset. The ECD of PM1 was estimated to be in area 4. And the ECDs of PM2 were estimated to be in area 4, SMA, and the PPC over the hemisphere contralateral to the movement, and in S2 of both hemispheres (Figure 2). The peak latency of each source activity was obtained in the range 54-109 $\mathrm{ms}$ in the SMA, 64-114 ms in the PPC, and 84-184 ms in the S2 (Figure 3).

\section{Cortical Excitability During passive Movements}

Several studies have reported consistent MEP changes in response to TMS at specific intervals following peripheral nerve electrical stimulation. For example, SAI was observed when the ISI between median nerve electrical stimulation and TMS was set at 20-50 ms ${ }^{79-12}$, whereas AF was observed with an ISI of $50-100 \mathrm{~ms}^{9,12-15)}$. It is possible that 


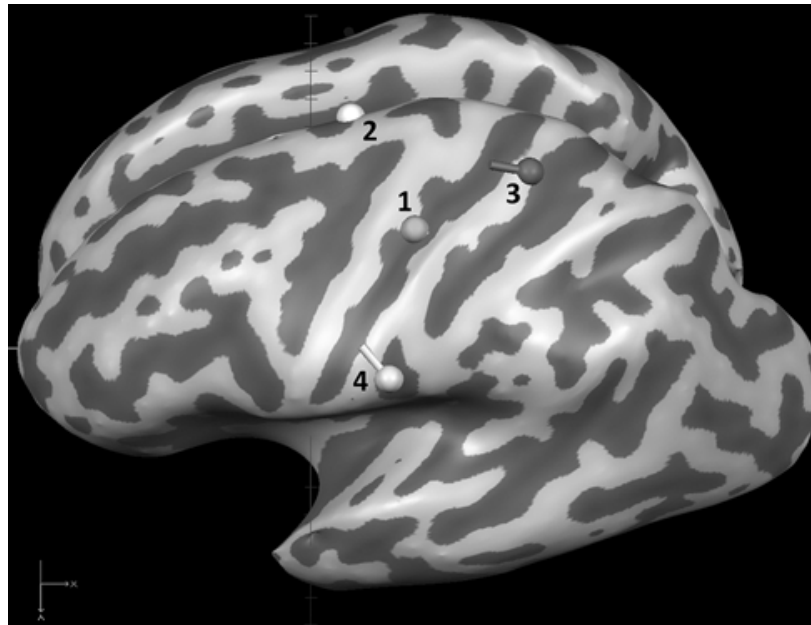

Figure 2.

Equivalent current dipoles (ECDs) following passive movement overlapped on the inflated brain of a representative subject. In this subject, the ECDs were estimated at the primary sensorimotor area (dipole 1), supplementary motor area (SMA, dipole 2), posterior parietal cortex (PPC, dipole 3), and contralateral secondary somatosensory cortex (cS2, dipole 4). (Onishi et al., 201329) ) passive movement could affect M1 excitability by activating afferent inputs according to the direction of movement. Unlike electrical peripheral stimulation paired with TMS, there have been no studies on SAI or AF during passive movement, so we conducted a study to elucidate the effects of joint angle and passive movement direction on M1 excitability $^{30)}$. This showed that M1 excitability increased 125 ms after the start of passive movements, suggesting that AF was evoked by somatosensory input with passive movements $^{30)}$. We also investigated whether M1 excitability depended on the ISI between the passive movement and the TMS pulse and whether modulation of M1 excitability depended on the movement velocity $\left(40^{\circ} / \mathrm{s}\right.$ and $\left.160 \% \mathrm{~s}\right)$ and joint angle of the passive movement ${ }^{31}$. Hence, the index finger was passively moved in the adduction direction (Figure 4) followed by TMS pulses in the midrange of the metacarpophalangeal joint with ISIs of 30, 60, 90, 120, 150, 180, and $210 \mathrm{~ms}$. The results showed that MEPs were significantly facilitated at 90, 120, and $150 \mathrm{~ms}$ without Fwave changes (Figure 5), and that MEP facilitation depended on the movement velocity (Figure 6) and joint angle (Figure 7). In addition, MEP significantly decreased at 30 a)



b)

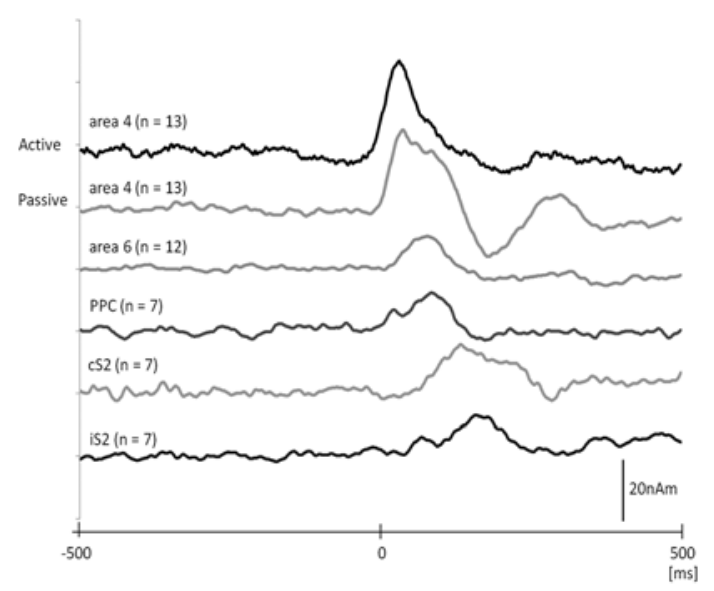

c)

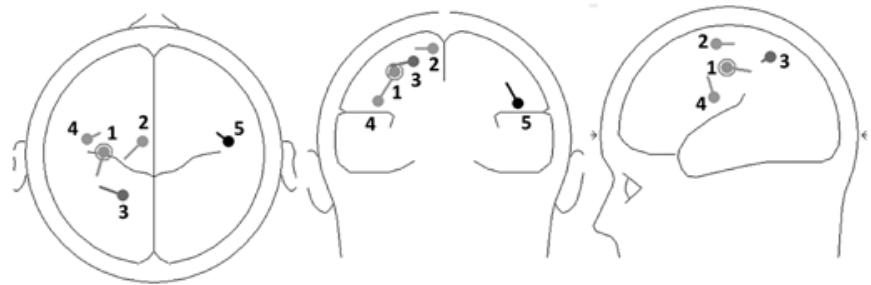

Figure 3.

Time courses of source activity and the locations of sources using Brain Electrical Source Analysis. a) Time course for each source activity in all subjects. b) Time course of the averaged source activity of each source. c) Schematic presentation of the locations of all the dipoles following passive movement. Area 4 (n $=13$ ); area 6 (supplementary motor area, SMA, $\mathrm{n}=12$ ); posterior parietal cortex $(\mathrm{PPC}, \mathrm{n}=7)$; contralateral secondary somatosensory cortex $(\mathrm{cS} 2, \mathrm{n}=7)$; ipsilateral secondary somatosensory cortex (iS2, $\mathrm{n}=7$ ). (Onishi et al., 201329) ) 


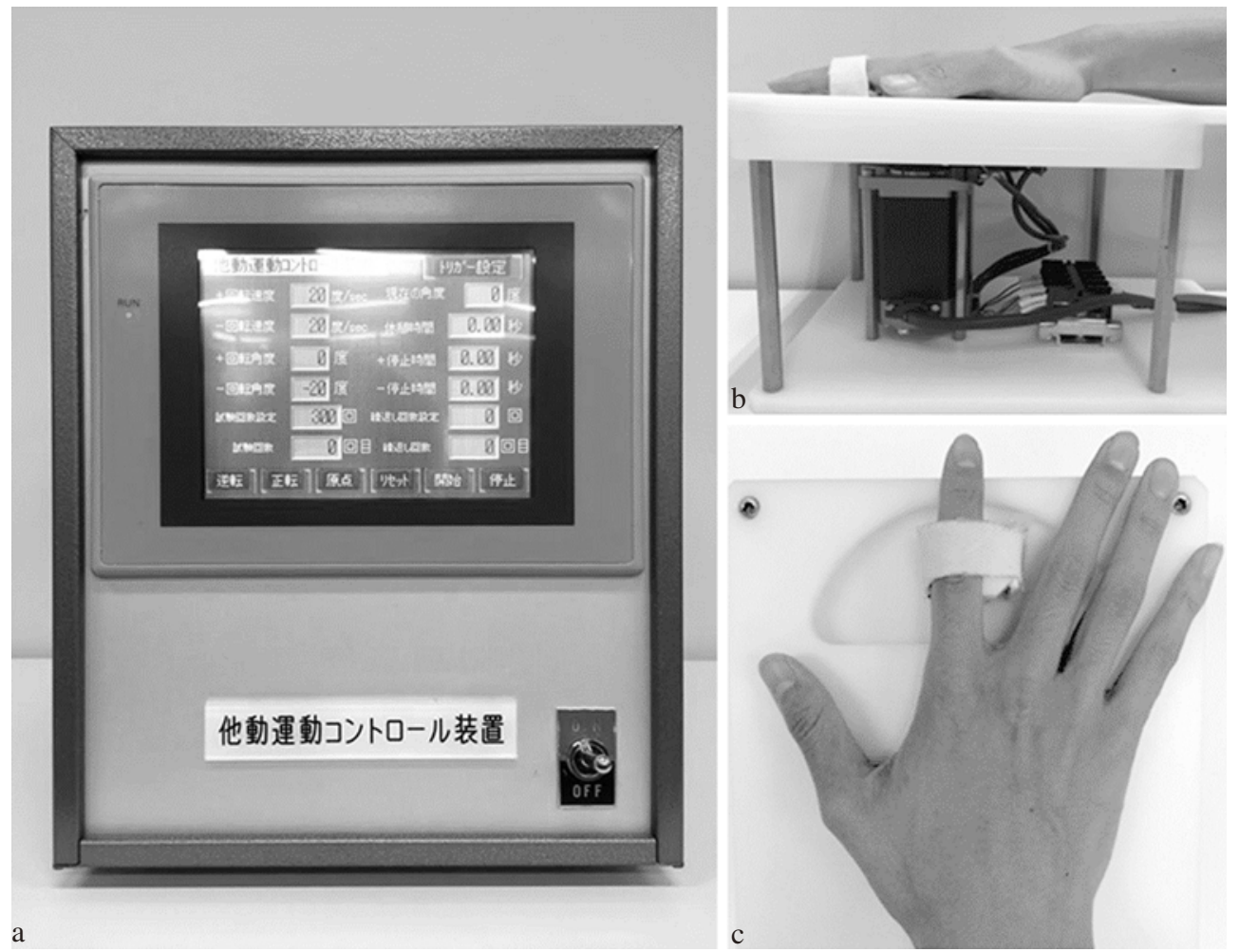

Figure 4.

The passive movement control apparatus. a) The main device controls the velocity and range of the movement. b) c) The secondary device produces repetitive passive movements of the right index finger. (Sasaki, et al., 201749) )

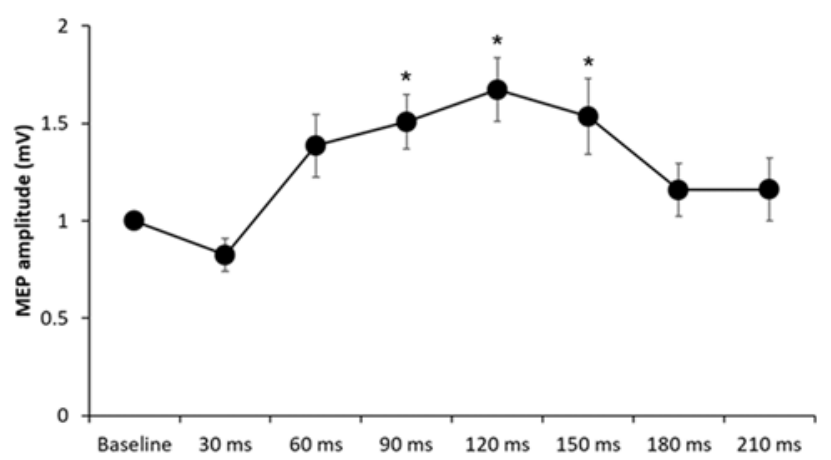

Figure 5.

The change in the amplitude of motor-evoked potentials (MEPs) with the inter-stimulus interval (ISI). The data show mean \pm standard error MEP amplitudes ( $n=20)$. Passive movements of the index finger for the adduction direction were performed at a velocity of $80 \%$. Transcranial magnetic stimulation (TMS) pulses followed the passive movements with ISIs of 30, 60, 90, 120, 150, 180, and $210 \mathrm{~ms}$. The TMSs were performed for the left M1, and the MEPs were recorded when the index finger was at the intermediate position. ${ }^{*} \mathrm{p}<0.05$ compared with baseline. (Sasaki, et al., 201731) )

ms of ISI only under $160^{\circ} / \mathrm{s}$ velocity. Thus, these experiments demonstrated that the facilitation of M1 excitability by somatosensory inputs induced by passive movements depended on the ISIs, the passive movement velocity, and the joint angle.

\section{Cortical Excitability after Repetitive Passive Movements}

In the field of rehabilitation, repetitive voluntary movements or passive movements are widely used to enhance muscle strength, to improve range of motion, and to promote motor learning or motor function in patients, such as for those who have suffered a stroke. Corticospinal excitability temporarily declines after voluntary exercise, a phenomenon referred to as post-exercise cortical depression $(\mathrm{PED})^{32-37)}$. A number of studies have reported that PED persisted for 20-30 min after exhaustive exercise at maximum voluntary contraction ${ }^{35,37,38)}$, and that it can be induced by light repetitive voluntary movement ${ }^{39-43)}$. In our own study, we showed that PED was induced by index finger abduction movement at $10 \%$ maximum voluntary contraction at a rate of $0.5 \mathrm{~Hz}$ for $10 \mathrm{~min}^{41)}$, and Teo et al. ${ }^{42)}$ observed PED that persisted for $8 \mathrm{~min}$ after $10 \mathrm{~s}$ of non-exhaustive finger flexion-extension movement. These observations demonstrate that both exhaustive and non-exhaustive movement can induce PED.

During the period of PED, the F-wave amplitude remains stable, indicating that spinal excitability does not 


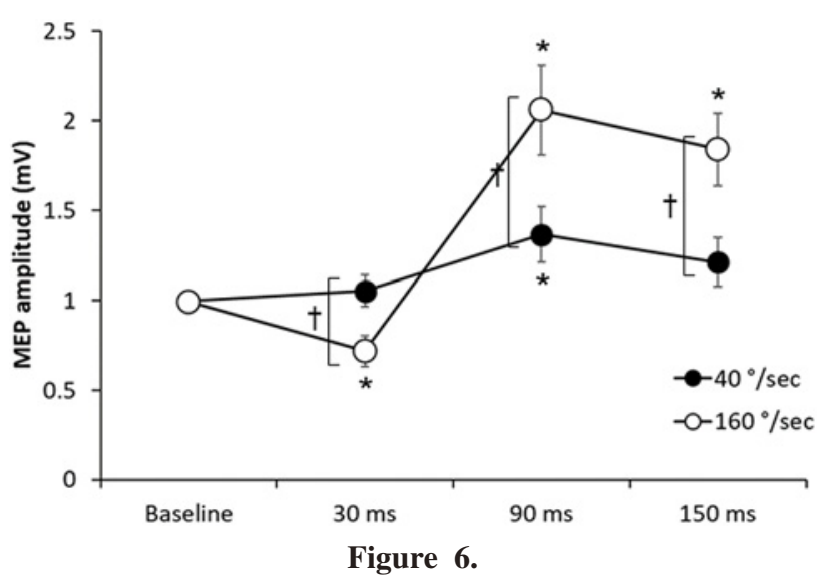

The effect of movement velocity on motor-evoked potential (MEP) amplitudes. Passive movements of the index finger in the adduction direction were performed at velocities of $40 \% \mathrm{~s}$ and $160 \%$ s. Inter-stimulus intervals (ISIs) were set at 30, 90, and $150 \mathrm{~ms}$, and the MEPs were recorded with the finger in the intermediate position. Black and white circles indicate the mean \pm standard error MEP amplitudes for all subjects $(n=15)$ for each ISI at velocities of $40 \%$ and $160 \%$, respectively. The MEPs differed significantly between $40 \%$ and $160 \%$ s for each ISI. At $40 \%$, there was a significant increase in MEP only at an ISI of $90 \mathrm{~ms}$; but at $160 \%$ s, the MEP was significantly lower than baseline at $30 \mathrm{~ms}$ and significantly higher at 90 and $120 \mathrm{~ms}$. ${ }^{*} \mathrm{p}$ $<0.05$ compared with baseline. $\dagger p<0.05$ for the difference between the two velocities. (Sasaki, et al., 201731)

change ${ }^{35,44)}$, whereas inhibitory cortical circuit transmission increases, as shown by increased SICI $^{42,43}$. It is therefore thought that PED results from inhibition of the excitability of M1. In addition, PED fluctuates according to the frequency of voluntary movement and the strength and type of muscle contraction ${ }^{39,42,45)}$. Potential mechanisms for PED include cortical depression, decreased neurotransmitter levels, the decreased excitability of intracortical glutamatergic networks, and the increased excitability of inhibitory GABAergic networks.

There have been inconsistent results for how M1 excitability changes after passive exercise, with studies re-

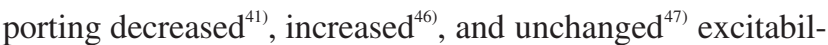
ity. Miyaguchi et al. ${ }^{41}$ ) reported that repetitive passive movement (RPM) of the index finger for $10 \mathrm{~min}$ at $0.5 \mathrm{~Hz}$ reduced M1 excitability, whereas Mace et al. ${ }^{46)}$ reported that RPM of the wrist for $60 \mathrm{~min}$ at an average frequency of 1.0 $\mathrm{Hz}$ increased M1 excitability, and Lotze et al. and McDonnell et al. observed no changes in MEPs after RPM for 30 $\min ^{47,48)}$. Table 1 summarizes six reports of studies that investigated MEP changes associated with passive movements. The differences in M1 excitability between studies may have been influenced by differences in various stimuli, such as the duration of movement, speed of movement, presence or absence of a duty cycle of repeated movement and rest, and the degree of active attention given to the

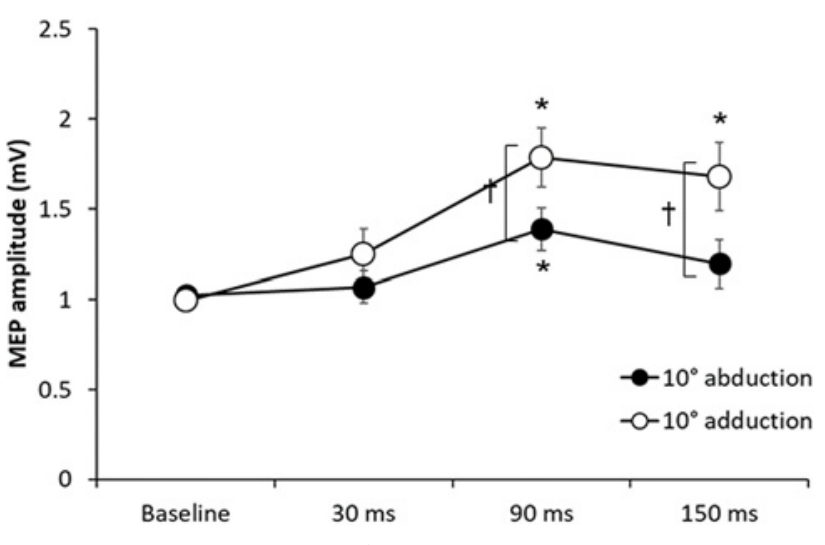

Figure 7.

The effect of joint angle on motor-evoked potential (MEP) amplitudes. Passive movements of the index finger in the adduction direction was performed at a velocity of $80 \%$ s. Inter-stimulus intervals (ISIs) were set at 30, 90, and $150 \mathrm{~ms}$, and MEPs were recorded with the finger in the $10^{\circ}$ abduction or $10^{\circ}$ adduction position (shortening vs. extension of the first dorsal interosseous muscle). Black and white circles show the mean \pm standard error MEP amplitudes for all subjects $(n=15)$ in the $10^{\circ}$ abduction position and $10^{\circ}$ adduction, respectively, for each ISI. The MEPs were significantly different between the $10^{\circ}$ abduction and $10^{\circ}$ adduction positions at 90 and $150 \mathrm{~ms}$. For $10^{\circ}$ abduction, the MEP was significantly higher than baseline only at 90 $\mathrm{ms}$; for $10^{\circ}$ adduction, the MEP was significantly higher at 90 and $150 \mathrm{~ms}$. ${ }^{*} \mathrm{p}<0.05$ compared with baseline. $\dagger \mathrm{p}<0.05$ for the difference between the two joint angles. (Sasaki, et al., 201731))

movement by the participant.

To clarify the factors that influence M1 excitability, we first investigated the effect of passive movement speed on PED after 10 min of RPM ${ }^{49}$. We applied RPMs of different frequencies to examine whether movement frequency contributed to the modulation of M1 excitability. The right index finger was passively abducted and adducted for 10 min at $0.5,1.0,3.0$, and $5.0 \mathrm{~Hz}$. We confirmed movement frequency and joint angle during RPM for all subjects using a electrogoniometer attached to the metacarpophalangeal joint of right index finger (Figure $8 \mathrm{~A}-\mathrm{D}$ ). Background EMG was monitored from the right FDI muscle during RPM to confirm passive movement (i.e., no EMG activity) (Figure 8E). RPMs at $0.5 \mathrm{~Hz}$ and $1.0 \mathrm{~Hz}$ both resulted in MEPs decreased relative to baseline for $2 \mathrm{~min}$, whereas 5.0Hz RPM reduced MEPs for 15 min; however, 3.0-Hz RPM resulted in no change in MEPs (Figure 9). No F-wave changes were observed following any RPM intervention. We then used the paired-pulse TMS technique to investigate whether RPM modulated the cortical inhibitory circuit. We measured SICI before and after 1.0, 3.0, and 5.0-Hz RPM using paired-pulse TMS with an ISI of $3 \mathrm{~ms}$. Both $1.0-\mathrm{Hz}$ and $5.0-\mathrm{Hz}$ RPM resulted in an increase in SICI compared with baseline. These results suggest that M1 ex- 
Table 1. Summary of studies investigating changes in motor-evoked potentials (MEP) after repetitive passive movements.

\begin{tabular}{|c|c|c|c|c|c|c|c|c|}
\hline & $\begin{array}{l}\text { duration } \\
\text { (min) }\end{array}$ & $\begin{array}{l}\text { number of } \\
\text { movements }\end{array}$ & $\begin{array}{c}\text { velosity } \\
\text { (degree/sec) }\end{array}$ & $\begin{array}{c}\text { joint of } \\
\text { movement }\end{array}$ & $\begin{array}{l}\text { range of } \\
\text { movement }\end{array}$ & $\begin{array}{l}\text { duty } \\
\text { cycle }\end{array}$ & attention & MEP \\
\hline Mace 46$)$ & 60 & 1800 & 120 & wrist & $-45 \Leftrightarrow 45$ & + & + & $\uparrow$ \\
\hline Lotze ${ }^{47)}$ & 30 & 300 & 309 & wrist & $0 \Leftrightarrow 55$ & + & + & $\rightarrow$ \\
\hline Miyaguchi41) & 10 & 300 & 20 & index & $0 \Leftrightarrow 20$ & - & - & $\downarrow$ \\
\hline \multirow[t]{4}{*}{ Sasaki ${ }^{49)}$} & 10 & 300 & 20 & index & $0 \Leftrightarrow 20$ & - & - & $\downarrow$ \\
\hline & 10 & 600 & 40 & index & $0 \Leftrightarrow 20$ & - & - & $\downarrow$ \\
\hline & 10 & 1800 & 120 & index & $0 \Leftrightarrow 20$ & - & - & $\downarrow$ \\
\hline & 10 & 3000 & 200 & index & $0 \Leftrightarrow 20$ & - & - & $\downarrow$ \\
\hline \multirow[t]{4}{*}{ Otsuka ${ }^{53)}$} & 10 & 300 & 15 & index & $-15 \Leftrightarrow 0$ & - & - & $\downarrow$ \\
\hline & 10 & 300 & 15 & index & $0 \Leftrightarrow 15$ & - & - & $\downarrow$ \\
\hline & 10 & 150 & 15 & index & $-15 \Leftrightarrow 15$ & - & - & $\downarrow$ \\
\hline & 10 & 300 & 15 & index & $15 \Leftrightarrow 30$ & - & - & $\downarrow$ \\
\hline \multirow[t]{3}{*}{ Tsuiki $^{56)}$} & 10 & 600 & 40 & index & $0 \Leftrightarrow 20$ & - & - & $\downarrow$ \\
\hline & 10 & 240 & 40 & index & $0 \Leftrightarrow 20$ & + & - & $\rightarrow$ \\
\hline & 10 & 600 & 100 & index & $0 \Leftrightarrow 20$ & + & - & $\downarrow$ \\
\hline
\end{tabular}

Notes: "Index" refers to index finger. Duty cycle + and - refer to the presence or absence, respectively, of a duty cycle including rest periods. Attention + and - refer to attention being given or not given, respectively, to the joint being moved. The arrows $\uparrow, \downarrow$, and $\rightarrow$ indicate increased, decreased, or unchanged MEPs, respectively.

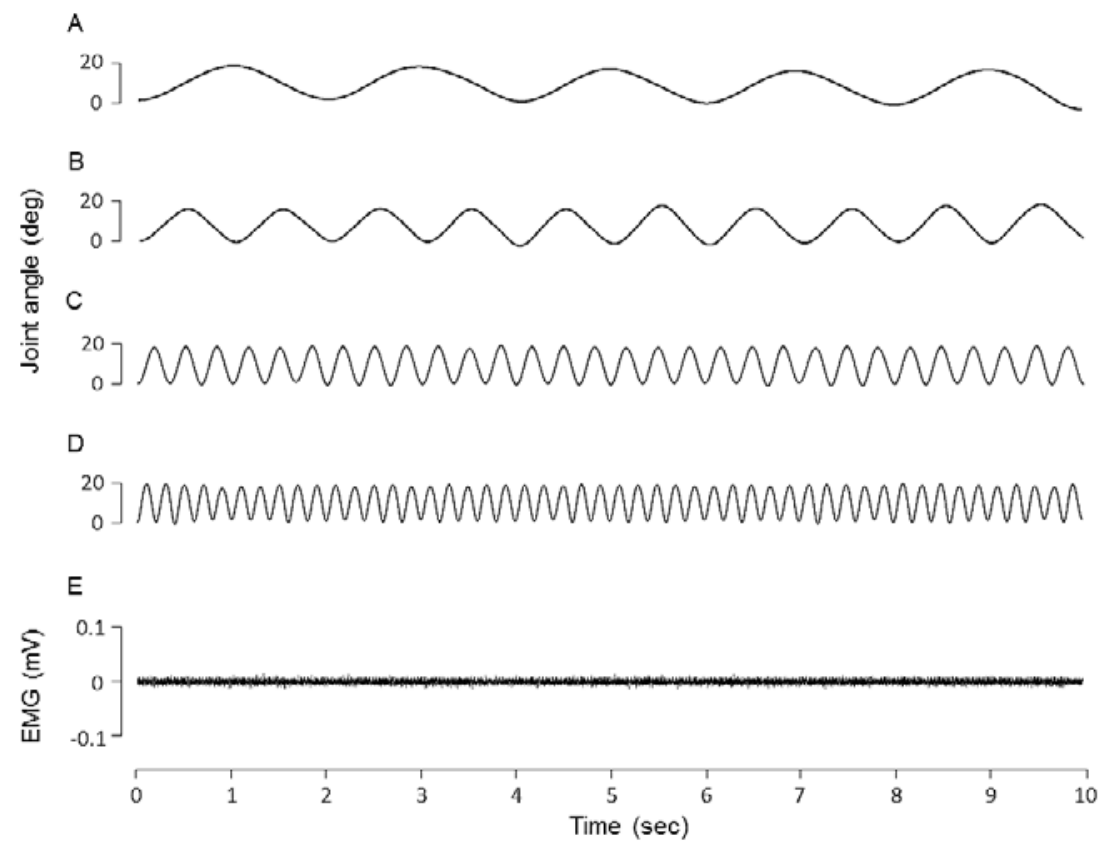

Figure 8.

Kinematic measurements during repetitive passive movement of the right index finger. (A) Joint angle during 0.5 Hz-RPM, (B) Joint angle during 1.0 Hz-RPM, (C) Joint angle during 3.0 Hz-RPM, (D) Joint angle during 5.0 Hz-RPM, (E) EMGs of the FDI muscle during 5.0 Hz-RPM. (Sasaki, et al., 201749) )

citability decreases after RPM in a manner that depends on the movement frequency, possibly through frequencydependent enhancement of the cortical inhibitory circuit in M1. However, those experiments were not able to clarify why the MEP depression and SICI increase were not observed specifically at $3.0-\mathrm{Hz}$ RPM. We therefore investi- gated whether RPM affected primary somatosensory cortex (S1) excitability ${ }^{50)}$. Somatosensory evoked potentials were recorded after 1.0, 3.0, and 5.0-Hz RPM for $10 \mathrm{~min}$. Only the $3.0-\mathrm{Hz}$ RPM resulted in a decrease in the $\mathrm{P} 45$ component of the somatosensory evoked potentials. Because theta burst stimulation over S1 suppresses S1 excitability and fa- 


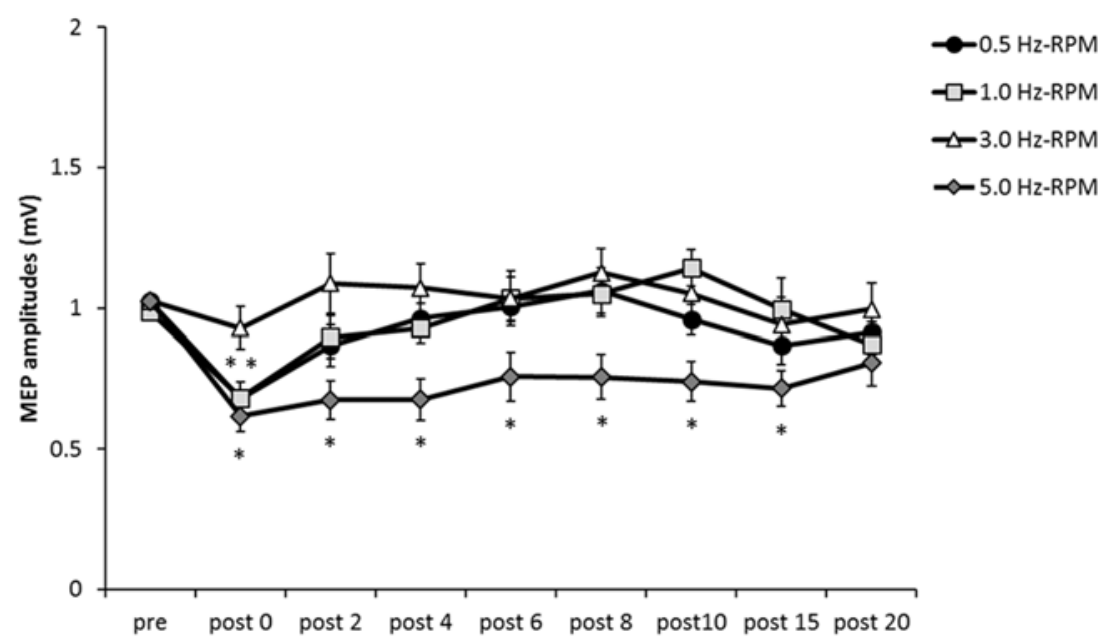

Figure 9.

The effect of the frequency of rapid passive movement (RPM) on MEP amplitudes. Time course of change in mean \pm standard error MEP amplitudes for all subjects ( $\mathrm{n}$ $=15$ ) following $0.5,1.0,3.0$, and 5.0-Hz RPM. $* \mathrm{p}<0.05$ compared with the pre value. (Sasaki, et al., 201749) )

a)

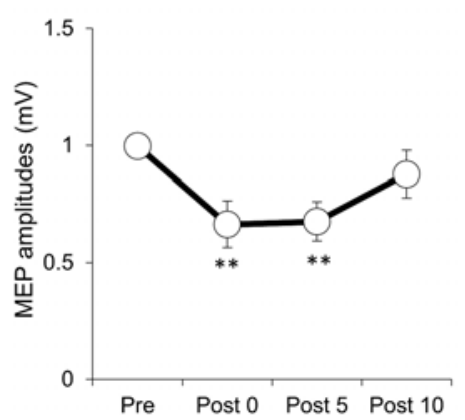

b)

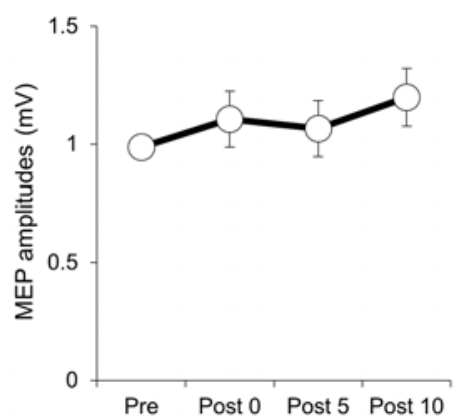

c)

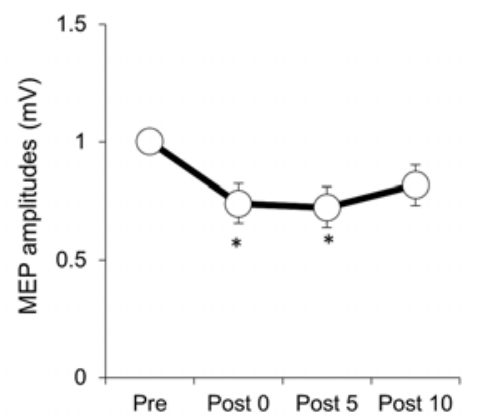

Figure 10.

Motor-evoked potential (MEP) amplitudes before and after repetitive passive movements (RPMs) under three conditions. a) Continuous RPM (600 movements); the MEP amplitude decreased significantly at Post0 and Post-5 compared with Pre. b) Intermittent passive movement (240 movements); there were no significant differences in MEP amplitude between before and after the passive movements. c) Intermittent passive movement (600 movements); the MEP amplitude decreased significantly at Post -0 and Post -5 compared with Pre. $* * \mathrm{p}<0.01$ compared with the pre value. $* \mathrm{p}<0.05$ compared with the pre value.

cilitates M1 excitability ${ }^{51,52)}$, it is possible that the depression in S1 excitability after the 3.0-Hz RPM may enhance M1 excitability through the nerve fibers from S1 to M1, thereby resulting in PED being disturbed by the 3.0-Hz RPM.

We next examined the influence on PED of the range of passive movement (the extension amplitude of the muscle $)^{53)}$. The index finger was passively moved from $15^{\circ} \mathrm{ab}-$ duction to $15^{\circ}$ adduction, $15^{\circ}$ abduction to $0^{\circ}, 0^{\circ}$ to $15^{\circ}$ adduction, and $15^{\circ}$ adduction to $30^{\circ}$ adduction, with each movement at $15 \%$ s for $10 \mathrm{~min}$. MEPs and F-waves were measured before and after each RPM. The amplitude of MEPs significantly decreased after all the RPMs, but the Fwave amplitude remained stable. These results suggest that the range of passive movement does not markedly influence the magnitude of PED.

A previous study reported that corticospinal excitability increased after passive movements for $60 \mathrm{~min}$ with a duty cycle that included 5-8 s of rest after every 10 movements ${ }^{46)}$. In another study that used peripheral electric stimulation, intermittent stimulation with a duty cycle of repeated stimulation and rest at an intensity above the motor threshold resulted in significantly increased corticospinal excitability $^{54)}$. It has also been shown that corticospinal excitability significantly decreased with continuous theta burst stimulation but increased when the theta burst stimulation was intermittent ${ }^{55}$. These findings suggest that con- 
tinuous and intermittent interventions with duty cycles of repeated stimulus and rest may have different effects on corticospinal excitability. We therefore examined the effect on M1 excitability of the presence or absence of a duty cycle for the $\mathrm{RPM}^{56)}$. Repetitive passive abduction-adduction movements of the right index finger from a neutral position to $20^{\circ}$ of abduction were performed for $10 \mathrm{~min}$ under three conditions: 1 , movement velocity of $40 \%$ s and continuous RPM (600 movements); 2, movement velocity of $40^{\circ} / \mathrm{s}$ and intermittent RPM (240 movements), with a configured duty cycle of $4 \mathrm{~s}$ on/6 s off; and 3, movement velocity of $100 \%$ and intermittent RPM (600 movements), with a configured duty cycle of $4 \mathrm{~s}$ on/ $6 \mathrm{~s}$ off. M1 excitability was significantly reduced under conditions 1 and 3 (Figure 10). These results suggest that changes in corticospinal excitability do not depend on the presence or absence of the duty cycle but are influenced by the number of movements.

Attention is closely related to cortical excitability. For example, during paired associative stimulation interventions, M1 excitability increased significantly when attention was directed to the stimulation side, but there was no change when focusing on the other hand ${ }^{57)}$. It has also been reported that SICI decreases and M1 excitability increased when attention was paid to the target hand during finger movements $^{58)}$, repetitive $\mathrm{TMS}^{59)}$, or vibration stimulation ${ }^{60)}$, but with no change in M1 excitability when there was no attention on the hand. These findings suggested that attention to the stimulated side during an intervention diminishes the activity of the suppressive circuits in the cortex, thereby increasing corticospinal excitability. We therefore investigated the influence on M1 excitability of paying attention to passive movement and found that, when attention was paid to the moving finger during passive movement, corticospinal excitability increased, whereas corticospinal excitability did not change under conditions where no attention was directed to the passive finger movements (under review).

\section{Conclusion}

In this review, we summarized cortical activity and excitability associated with passive movement, focusing in particular on cortical excitability after RPM. Corticospinal excitability is thought to be influenced by various factors, such as the duration and velocity of movements, the presence or absence of a duty cycle of repeated movement and rest, and whether active attention was directed at the movement. We therefore performed several experiments involving RPMs, which showed that, whether or not there was a duty cycle of repeated movement and rest, RPM resulted in a temporary decrease in cortical excitability when no attention was paid to the passive movement, but an increase in cortical excitability when attention was directed at the movement.
Acknowledgments: This work was supported by a Grant-in-Aid for Scientific Research (B) 16H03207 from the Japan Society for the Promotion of Science. In addition, the author would like to thank Enago Inc. (http://www. enago.jp/) for editorial assistance with the manuscript.

Conflict of Interest: The author declares no conflicts of interest.

\section{References}

1) Kujirai T, Caramia MD, et al.: Corticocortical inhibition in human motor cortex. J Physiol. 1993; 471: 501-519.

2) Ziemann U, Reis J, et al.: Tms and drugs revisited 2014. Clin Neurophysiol. 2015; 126: 1847-1868.

3) Ziemann U: Tms and drugs. Clin Neurophysiol. 2004; 115 : 1717-1729.

4) Di Lazzaro V, Pilato F, et al.: Segregating two inhibitory circuits in human motor cortex at the level of gabaa receptor subtypes: A tms study. Clin Neurophysiol. 2007; 118: 2207-2214.

5) Di Lazzaro V, Pilato F, et al.: Gabaa receptor subtype specific enhancement of inhibition in human motor cortex. J Physiol. 2006; 575: 721-726.

6) Ilic TV, Meintzschel F, et al.: Short-interval paired-pulse inhibition and facilitation of human motor cortex: The dimension of stimulus intensity. J Physiol. 2002; 545: 153-167.

7) Tokimura H, Di Lazzaro V, et al.: Short latency inhibition of human hand motor cortex by somatosensory input from the hand. $\mathrm{J}$ Physiol. 2000; 523 Pt 2: 503-513.

8) Turco CV, El-Sayes J, et al.: Short- and long-latency afferent inhibition; uses, mechanisms and influencing factors. Brain Stimul. 2018; 11: 59-74.

9) Devanne H, Degardin A, et al.: Afferent-induced facilitation of primary motor cortex excitability in the region controlling hand muscles in humans. Eur J Neurosci. 2009; 30: 439-448.

10) Kotb MA, Mima T, et al.: Effect of spatial attention on human sensorimotor integration studied by transcranial magnetic stimulation. Clin Neurophysiol. 2005; 116: 1195-1200.

11) Tamburin S, Fiaschi A, et al.: Sensorimotor integration to cutaneous afferents in humans: The effect of the size of the receptive field. Exp Brain Res. 2005; 167: 362-369.

12) Kojima $S$, Onishi $H$, et al.: No relation between afferent facilitation induced by digital nerve stimulation and the latency of cutaneomuscular reflexes and somatosensory evoked magnetic fields. Front Hum Neurosci. 2014; 8: 1023.

13) Degardin A, Devos D, et al.: Deficit of sensorimotor integration in normal aging. Neurosci Lett. 2011; 498: 208-212.

14) Deletis V, Schild JH, et al.: Facilitation of motor evoked potentials by somatosensory afferent stimulation. Electroencephalography \& Clinical Neurophysiology. 1992; 85: 302-310.

15) Komori $\mathrm{T}$, Watson $\mathrm{BV}$, et al.: Influence of peripheral afferents on cortical and spinal motoneuron excitability. Muscle \& Nerve. 1992; 15: 48-51.

16) Di Lazzaro V, Oliviero A, et al.: Muscarinic receptor blockade has differential effects on the excitability of intracortical circuits 
in the human motor cortex. Exp Brain Res. 2000; 135: 455-461.

17) Di Lazzaro V, Oliviero A, et al.: Effects of lorazepam on short latency afferent inhibition and short latency intracortical inhibition in humans. J Physiol. 2005; 564: 661-668.

18) Mima T, Terada K, et al.: Somatosensory evoked potentials following proprioceptive stimulation of finger in man. Exp Brain Res. 1996; 111: 233-245.

19) Reddy H, Floyer A, et al.: Altered cortical activation with finger movement after peripheral denervation: Comparison of active and passive tasks. Exp Brain Res. 2001; 138: 484-491.

20) Weiller C, Juptner $M$, et al: : Brain representation of active and passive movements. Neuroimage. 1996; 4: 105-110.

21) Alary F, Doyon B, et al.: Event-related potentials elicited by passive movements in humans: Characterization, source analysis, and comparison to fmri. Neuroimage. 1998; 8: 377-390.

22) Radovanovic S, Korotkov A, et al:: Comparison of brain activity during different types of proprioceptive inputs: A positron emission tomography study. Exp Brain Res. 2002; 143: 276-285.

23) Terumitsu M, Ikeda K, et al.: Participation of primary motor cortex area $4 \mathrm{a}$ in complex sensory processing: 3.0-t fmri study. Neuroreport. 2009; 20: 679-683.

24) Alary F, Simoes C, et al: : Cortical activation associated with passive movements of the human index finger: An meg study. Neuroimage. 2002; 15: 691-696.

25) Druschky K, Kaltenhauser M, et al.: Somatosensory evoked magnetic fields following passive movement compared with tactile stimulation of the index finger. Exp Brain Res. 2003; 148: 186-195.

26) Lange $\mathrm{R}$, Nowak $\mathrm{H}$, et al: : Passive finger movement evoked fields in magnetoencephalography. Exp Brain Res. 2001; 136: 194-199.

27) Woldag H, Waldmann G, et al.: Cortical neuromagnetic fields evoked by voluntary and passive hand movements in healthy adults. J Clin Neurophysiol. 2003; 20: 94-101.

28) Xiang J, Hoshiyama M, et al.: Somatosensory evoked magnetic fields following passive finger movement. Brain Res Cogn Brain Res. 1997; 6: 73-82.

29) Onishi H, Sugawara K, et al.: Neuromagnetic activation following active and passive finger movements. Brain Behav. 2013; 3 : 178-192.

30) Nakagawa M, Sasaki R, et al.: Effects of passive finger movement on cortical excitability. Front Hum Neurosci. 2017; 11: 216.

31) Sasaki R, Tsuiki S, et al.: Somatosensory inputs induced by passive movement facilitate primary motor cortex excitability depending on the interstimulus interval, movement velocity, and joint angle. Neuroscience. 2018; 386: 194-204.

32) Lewis GN, Byblow WD, et al.: Phasic modulation of corticomotor excitability during passive movement of the upper limb: Effects of movement frequency and muscle specificity. Brain Res. 2001; 900: 282-294.

33) Sacco P, Thickbroom GW, et al.: Changes in corticomotor excitability after fatiguing muscle contractions. Muscle \& Nerve. 2000; 23: 1840-1846.
34) Samii A, Wassermann EM, et al.: Characterization of postexercise facilitation and depression of motor evoked potentials to transcranial magnetic stimulation. Neurology. 1996; 46: 13761382.

35) Zanette G, Bonato C, et al.: Long-lasting depression of motorevoked potentials to transcranial magnetic stimulation following exercise. Exp Brain Res. 1995; 107: 80-86.

36) Brasil-Neto JP, Pascual-Leone A, et al.: Postexercise depression of motor evoked potentials: A measure of central nervous system fatigue. Exp Brain Res. 1993; 93: 181-184.

37) McKay WB, Tuel SM, et al.: Focal depression of cortical excitability induced by fatiguing muscle contraction: A transcranial magnetic stimulation study. Exp Brain Res. 1995; 105: 276-282.

38) Liepert J, Kotterba S, et al.: Central fatigue assessed by transcranial magnetic stimulation. Muscle \& Nerve. 1996; 19: 14291434.

39) Miyaguchi S, Kojima S, et al.: Do differences in levels, types, and duration of muscle contraction have an effect on the degree of post-exercise depression? Front Hum Neurosci. 2016; 10: 159.

40) Miyaguchi S, Kojima S, et al.: Decrease in short-latency afferent inhibition during corticomotor postexercise depression following repetitive finger movement. Brain Behav. 2017; 7: e00744.

41) Miyaguchi S, Onishi H, et al.: Corticomotor excitability induced by anodal transcranial direct current stimulation with and without non-exhaustive movement. Brain Res. 2013; 1529: 83-91.

42) Teo WP, Rodrigues JP, et al.: Post-exercise depression in corticomotor excitability after dynamic movement: A general property of fatiguing and non-fatiguing exercise. Exp Brain Res. 2012; 216: 41-49.

43) Teo WP, Rodrigues JP, et al.: Changes in corticomotor excitability and inhibition after exercise are influenced by hand dominance and motor demand. Neuroscience. 2012; 210: 110-117.

44) Bonato C, Zanette G, et al.: Cortical output modulation after rapid repetitive movements. Ital J Neurol Sci. 1994; 15: 489494.

45) Bonato C, Zanette G, et al.: Activity-dependent modulation of synaptic transmission in the intact human motor cortex revealed with transcranial magnetic stimulation. Cereb Cortex. 2002; 12: 1057-1062.

46) Mace MJ, Levin O, et al.: Corticospinal facilitation following prolonged proprioceptive stimulation by means of passive wrist movement. J Clin Neurophysiol. 2008; 25: 202-209.

47) Lotze M, Braun C, et al.: Motor learning elicited by voluntary drive. Brain. 2003; 126: 866-872.

48) McDonnell MN, Hillier SL, et al.: Continuous passive movement does not influence motor maps in healthy adults. Front Hum Neurosci. 2015; 9: 230.

49) Sasaki R, Nakagawa M, et al.: Regulation of primary motor cortex excitability by repetitive passive finger movement frequency. Neuroscience. 2017; 357: 232-240.

50) Sasaki R, Tsuiki S, et al.: Repetitive passive finger movement modulates primary somatosensory cortex excitability. Front Hum Neurosci. 2018; (In press).

51) Jacobs MF, Tsang P, et al.: $30 \mathrm{hz}$ theta-burst stimulation over 
primary somatosensory cortex modulates corticospinal output to the hand. Brain Stimul. 2014; 7: 269-274.

52) Tsang P, Jacobs MF, et al.: Continuous theta-burst stimulation over primary somatosensory cortex modulates short-latency afferent inhibition. Clin Neurophysiol. 2014; 125: 2253-2259.

53) Otsuka R, Sasaki R, et al.: Post-exercise cortical depression following repetitive passive finger movement. Neurosci Lett. 2017; 656: 89-93.

54) Chipchase LS, Schabrun SM, et al:: Corticospinal excitability is dependent on the parameters of peripheral electric stimulation: A preliminary study. Archives of Physical Medicine \& Rehabilitation. 2011; 92: 1423-1430.

55) Huang YZ, Edwards MJ, et al.: Theta burst stimulation of the human motor cortex. Neuron. 2005; 45: 201-206.

56) Tsuiki S, Sasaki R, et al.: Influence of passive finger movement with and without duty cycle on corticospinal excitability. Society for Neuroscience. Annual meeting (abstract). 2017.

57) Stefan K, Wycislo M, et al.: Modulation of associative human motor cortical plasticity by attention. J Neurophysiol. 2004; 92 : 66-72.

58) Thomson RH, Garry MI, et al.: Attentional influences on shortinterval intracortical inhibition. Clin Neurophysiol. 2008; 119: 52-62.

59) Conte A, Gilio F, et al.: Attention influences the excitability of cortical motor areas in healthy humans. Exp Brain Res. 2007; 182: 109-117.

60) Rosenkranz K and Rothwell JC: The effect of sensory input and attention on the sensorimotor organization of the hand area of the human motor cortex. J Physiol. 2004; 561: 307-320. 